\title{
El Límite Mioceno-Plioceno en el Pozo Cisne 1 (Golfo de México)
}

\section{Omaña Pulido, Lourdes* y Salazar Medina, Gisela* \\ INTRODUCCION}

El desarrollo de la exploración petrolera ha conducido a investigaciones cada vez más detalladas de los foraminíferos, y en las dos últimas décadas principalmente, se ha puesto énfasis en los planctónicos. Esto ha tenido como resultado el desarrollo de esquemas zonales basados sobre todo en la distribución de las especies y subespecies de foraminíferos planctónicos.

Recientemente se ha notado un creciente interés a nivel mundial sobre el límite Mioceno-Plioceno, lo que nos ha conducido a realizar una revisión detallada de la historia de dicho límite para entender mejor las observaciones hechas en el Pozo Cisne 1 .

\section{ANTECEDENTES HISTORICOS DEL LIMITE MIOCENO-PLIOCENO}

Lyell en 1833 (vol. 3) subdividió el Terciario basándose en sus propias investigaciones y en las del conquiólogo francés Deshayes. De un examen de cerca de 40,000 especímenes la proporción de especies vivientes a extintas fue aceptada como un caracter distintivo de las subdivisiones; los límites son como siguen:

Newer Pliocene: 90-95\%/o de especies aún vivientes.

Older Pliocene: más de 50\% de especies vivientes.
Miocene Period: 20-40\% $/ 0$ de especies vivientes Eocene Period: 0 a menos de 5\% de especies aún vivientes.

Lyell (op. cit.) consideró los afloramientos de Touraine, Bordeaux, Valle de Bormida y Superga cerca de Turín, como sedimentos del "Mioceno". Las formaciones marinas originalmente referidas a su "Older Pliocene" incluyeron localidades subapeninas del norte, como Parma, Asti, Siena, Perpignan, Niza y el English Crag. Para el "Newer Pliocene" introdujo posteriormente (1839) el término Pleistoceno.

El Mioceno y Plioceno fueron algunas veces unificadas bajo el nombre de Neoceno por los contemporáneos de Lyell, especialmente donde las divisiones no estaban bien diferenciadas.

En 1864 el geólogo Hörnes introdujo el término Neógeno para dichas edades (in Berggren 1971, p. 695).

Deben mencionarse para una mejor comprensión del límite Mioceno-Plioceno, algunos datos sobre los pisos que se han considerado sub y suprayaciendo al límite mencionado:

Messiniano.- Este término fue introducido en la literatura de una manera confusa por Mayer-Eymar en 1867 para los estratos regresivos de agua salobre que suprayacían al Tortoniano marino. Lo subdividió en tres partes:

1.- Estratos Billovitz.

2.- Estratos Inzersdorf. 


\section{3.- Estratos Eppelsheim (Austria).}

Refirió también una serie de unidades marinas en el Sur de Italia (Messina-Calabria) a su Messiniano.

Seguenza en 1868 propone el Piso Zancliano; pero Mayer-Eymar fue de la opinión que dichos estratos eran de la misma edad que su propio Messiniano, diciendo que el nombre Zancliano (de Zancla, el nombre griego de Messina antes de la conquista Romana) era demasiado clásico para su gusto, por lo que lo reemplazó con el nombre de Messiniano.

En su concepto original el Messiniano incluía las margas con ditomeas ("Trípoli"), evaporitas y la "Trubi" (lutitas de mar profundo con ricas faunas planctónicas). Seguenza $(1868,1879)$ restringió el Messiniano a la serie evaporítica y al "Trípoli". Consideró la unidad suprayaciente, la "Trubi", como Plioceno, e introdujo el término Zancliano para los sedimentos calcáreo-margosos con contenido fósil uniforme, pero excluyendo las arenas productoras de Amphistergina. De acuerdo a Seguenza el Zancliano representa un intervalo definido entre el Tortoniano y el Plioceno (Astiano-Piacenziano) y lo colocó en la base del Pliceno, el cual creyó pudiera ser dividido en tres partes: Zancliano, Piacenziano y Astiano.

Como consecuencia, el uso del término ha llevado a confusión, ya que ha sido aplicado variadamente a la serie de yeso-anhidrita ("Gessososolfifera") y a los depósitos de Edad Pontiano (Plioceno).

Debido a complicaciones tectónicas y sedimentológicas en la vecindad de Messina, cerca de Gesso, Sur de Italia, Selli (1960) ha seleccionado recientemente y descrito una sección neoestratotipo para el Piso Messiniano de Mayer-Eymar, la serie de Pasquasia-Capodarso en la provincia de Enna y Caltanissetta, en Sicilia central. La secuencia está limitada abajo por margas de Edad Tortoniano y arriba por la "Trubi", generalmente aceptada como representante del Plioceno basal $(=$ Zancliano) en esta área.

La subdivisión del Plioceno comienza con Rouville (1853, in Berggren, 1971, p. 758), quien creó el Piso Astiano para las arenas calcáreas amarillas de Asti en el Norte de Italia, correspondiendo exactamente al Plioceno más antiguo de Lyell ("Older Pliocene").

Mayer-Eymar (1858) propuso el nombre Pia- cenziano originalmente como un subpiso del Astiano, que incluye las facies arcillosas del Plioceno Inferior en el Norte de Italia.

Seguenza introdujo el término Zancliano para el Plioceno Inferior en 1868 teniendo como localidad tipo la región de Monte Peloritani en Sicilia. La Marga Trubi tiene una microfauna rica y diversificada que consiste de foraminiferos planctónicos y otros organismos pelágicos (nanofósiles).

Mayer-Eymar (1868) propuso el Tabianiano para las mangas azules que afloran en Tabiano Bagni, cerca de Salsomaggiore, en el norte de Italia, como el piso inferior del Plioceno. Las faunas de aguas más frías del Tabianiano no tienen los ricos elementos faunales escenciales para la correlación estratigráfica intercontinental.

Sin lugar a dudas fueron las faunas del Zancliano las que permitieron que Banner y Blow (1965) reconocieran su Zona N18 en el área Mediterránea y demostraran un traslape de los Pisos Piacenziano y Zancliano. El desarrollo de Sphaeroidinella dehiscens a partir de Sphaeroidinellopsis subdehiscens, así como el traslape de Globigerina nepenthes y $S$. dehiscens en la parte inferior del "Trubi" de Sicilia, ha sido considerado característico del Plioceno Inferior (Blow, 1969; Parker, 1967 cf. Blow, 1959 p. 196). Por esta razón el uso del Zancliano para el Plioceno Inferior es preferido al Tabianiano.

Recientemente, Cita (1975) propone un neoestratotipo para el límite Mioceno-Plioceno, sugiriendo que pudiera ser la base del Zancliano, representado por las Margas "Trubi" en Capo Rosello cerca de Realmonte (Agrigento, Sicilia). Corresponde a la primera aparición de condiciones permanentes de mar abierto en el Mediterráneo (o con el nacimiento del Neo Mediterráneo) posterior a la "crisis de salinidad" y desecación en el Mioceno Superior.

Desde el punto de vista bioestratigráfico el problema del limite Mioceno-Plioceno ha sido estudiado por Bandy (1963a, 1963b, 1964 y 1975) indicando que dicho límite coincide con la aparición evolutiva de Sphaeroidinella dehiscens (Parker y Jones). En 1967 Bandy y Wade ratificaron dicho concepto y mencionan que Parker (1964) reconoció el límite Mioceno-Plioceno en un estudio del Mohole (Este del Pacífico) en base al "datum" Sphaeroidinella dehiscens. Bandy y Casey (1969) confirman las observaciones anteriores.

Blow (1969) coloca el límite en la parte supe- 
rior de la Zona N18 por la aparición evolutiva de Sphaeroidinella dehiscens a partir de su ancestro Sphaeroidinellopsis subdehiscens paenedehiscens, señalando que es un "datum" facilmente reconocible para correlación mundial.

Berggren (1971) hace notar que el límite Mioceno-Plioceno no parece estar marcado por ningún criterio significativo entre los foraminíferos planctónicos. Sin embargo, la aparición de Sphaeroidinella dehiscens, en la base de la Zona N19, tiene lugar en el Plioceno Inferior y el traslape de la distribución estratigráfica de Globigerina nepenthes, Globorotalia tumida, G. multicamerata, G. miocenica $\vee$ Sphaeroidinellopsis subdehiscens interalia, puede ser usado para distinguir el Mioceno Superior del Plioceno Inferior.

Parker (1973) sitúa el "datum" Sphaeroidinella dehiscens después de la primera aparición de Globorotalia margaritae, estableciendo que $S$. dehiscens es separada como tal tan pronto como es visto un sólo especímen de Spaheroidinellopsis subdehiscens con una abertura suplementaria, más bien que cuando una parte sustancial de un conjunto es referible a éste.

Srnivas an y Srivastava (1974) indican que la aparición evolutiva de Sphaeroidinella dehiscens es un "datim" válido en las regiones tropicales y marca el límite Mioceno-Plioceno.

Stainforth et al (1975) no consideran el "datum" Sphaeroidinella para definir el límite,

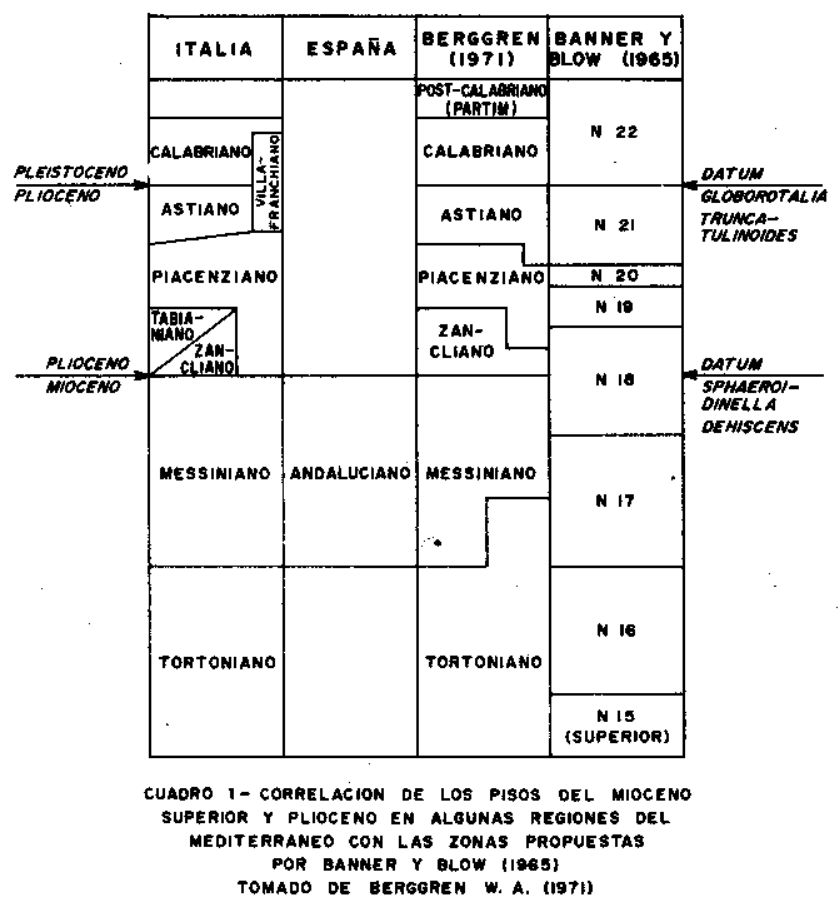

porque la aparición de una abertura suplementaria incipiente no es una base segura para definir un "datum" confiable. Ellos colocan el límite Mioceno-Plioceno utilizando el "datum" Globoratalia margaritae $y$ hacen notar que $S$. dehiscens no ocurre ni en Italia ni en otras partes tropicales hasta el Plioceno Superior.

Cita (1975) indica que el "datum" Sphaeroidinella es considerado un evento bioestratigráfico de primera clase que puede ser reconocido dentro de la Zona de Acmé de Sphaeroidinellopsis, la cual está presente en su neoestratotipo propuesto para el límite Mioceno-Plioceno en Sicilia.

El "datum" Sphaeroidinella ha sido utilizado también en Taiwan por Huang (1975) y Chang (1975) y en Japón por Asano, Takayanagi y Takayama (1975).

\section{EL LIMITE MIOCENO-PLIOCENO EN EL POZO. CISNE No. 1}

El Pozo Cisne No. 1 forma parte de un proyecto que se está realizando en la Sección de Micropaleontología del Cenozoico del Instituto Mexicano del Petróleo. El estudio de los foraminíferos planctónicos nos permitió reconocer el límite Mioceno-Plioceno, así como la identificación de dos zonas bioestratigráficas.

\section{LOCALIZACION DEL POZO ESTUDIADO}

El Pozo marino Cisne No. 1 se encuentra localizada aproximadamente a $18100 \mathrm{~m}$ al N, 600 E del Pozo Santa Ana No. 37.

Coordenadas Pemex: X 61769.42

Y 28962.67

Provincia de Huimanguillo, Comalcalco (Tabasco).

\section{BIOESTRATIGRAFIA}

Se describen a continuación dos zonas, una para el Mioceno Superior y otra del Plioceno.

\section{MIOCENO SUPERIOR}

\section{ZONA CON TURBOROTALIA ACOSTAEN-} SIS.

Autor: Bolli y Bermúdez, 1965, p. 130-131 (emend).

Esta Zona se caracteriza en nuestro material 


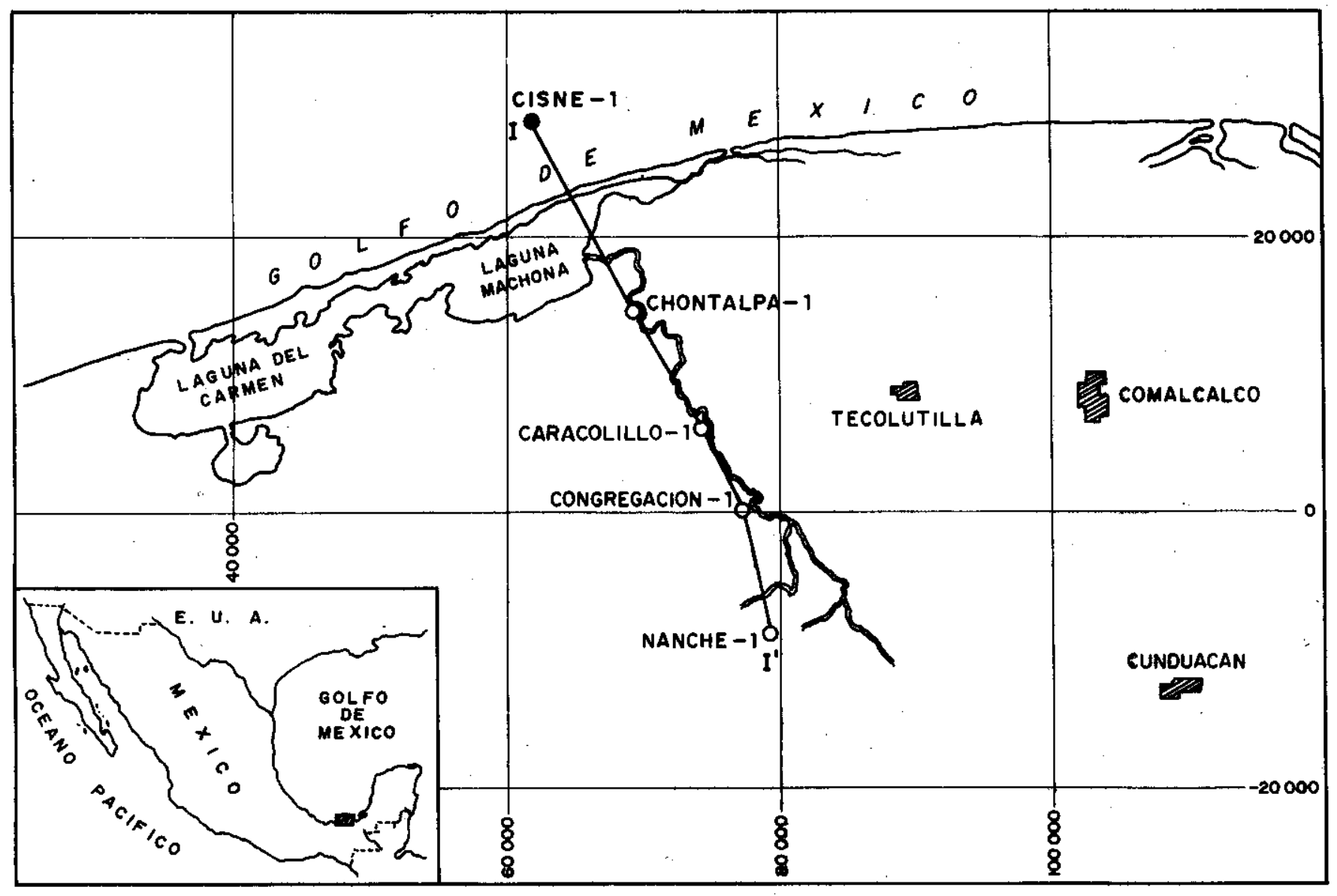

FIG. 1-LOCALIZACION DEL POZO CISNE - 1

ESC. 1: 400000

por la presencia de Turboratolia acostaensis Blow y Globorotalia margaritae Bolli y Bermúdez y la ausencia de Sphaeroidinelta dehiscens(Parker y Jones), cuya aparición evolutiva marcaría la cima de esta zona.

Se encuentran también:

Sphaeroidinellopsis subdehiscens Blow Sphaeroidinellopsis grimsdalei Keijzer Globigerinoides congabatus (Brady) Globigerinoides obliquus extremus Bolli y Bermudez

Globigerinoides obliquus obliquus,Bolli

Globigerinoides obliquss amplus Perconig

Globigerinoides ruber (d'Orbigny)

Globigerinoides trilobus ińmaturus LeRoy

Globigerinoides trilobus sacculifer (Brady)

Globigerinoides trilobus trilobus (Reuss)

Globigerina nepenthes Todd

Globigerina microstoma Cita, P. Silva y Rossi

Globigerina riveroae Bolli y Bermúdez
Turborofalia opima continuosa Blow

Turborotalia obesa Bolli

Globorotalia cultrata d'Orbigny

Globorotalia crassaformis Galloway y Wissler

Globorotalia menardii d'Orbigny

Globorotalia miocenica Palmer

Globoquadrina baroemoenensis LeRoy

Orbulina universa d'Orbigny

Orbulina suturalis Brönnimann

La Zona aquí estudiada es considerada como representativa del Mioceno Superior, porque Turborotalia acostaensis Blow es un microfósil utilizado para caracterizar este intervalo en diferentes partes del mundo (Blow, 1969; Berggren y Van Couvering, 1974; Haak y Postuma, 1975; Stainforth et al, 1975).

Esta zona se presenta en el Pozo Cisne No. 1 de 2200 a $4890 \mathrm{~m}$ de profundidad.

\section{PLIOCENO}

ZONA CON SPHAEROIDINELLA DEHIS- 
Autor: Blow, 1969, p. 254 (emend)

La Zona que a continuación se describe se caracteriza por la presencia abundante de Sphaeroidinella dehiscens (Parker y Jones) y Globorotalia ungulata Bermúdez.

La base de la Zona está marcada por la aparición evolutiva del fósil nominal.

Se encuentran además:

Glabigerinoides obliquus elongatus(d'Orbigny)

Globigerinoides obliquus extremus Bolli y Bermúdez

Globigerinoides obliquus amplus Perconig

Globigerinoides obliquus obliquus Bolli

Globigerinoides ruber (d'Orbigny)

Globigerinoides conglobatus (Brady)

Globigerinoides helicinus (d'Orbigny)

Sphaeroidinellopsis subdehiscens Blow

Sphaercidinellopsis grimsdalei Keijzer

Turborotalia humerosa Tokayanagi y Saito

Turborotalia acostaensis Blow

Turborotalia obesa Bolli

Globorotalia crassaformis Galloway y Wissler

Globorotalia cultrata d'Orbigny

Globorotalia miocenica Palmer

Globigerina nepenthes Todd

Globigerina decoraperta Takayanagi y Saito

Globigerina riveroae Bolli y Bermúdez

Globigerina juvenilis Bolli

Globigerina bulloides d'Orbigny

Globigerina falconensis Blow

Hastigerina aequilateralis (Brady)

Orbulina bilobata (d'Orbigny)

Orbulina saturalis Brönnimann

Orbulina universa d'Orbigny

A esta Zona se le asigna una Edad Plioceno por la presencia de Sphaeroidinella dehiscens; en fin, este microfósil es considerado por diferentes autores como Blow, 1969; Berggren y Van Couvering, 1974; Haak y Postuma, 1975; Stainforth et al, 1975 , para definir dicha edad.

La ausencia de Globorotalia truncatulinoides
d'Orbigny en los sedimentos estudiados, nos confirma la edad sugerida para este intervalo, porque la aparición evolutiva de este foraminífero es utilizada come un "datum" para marcar el límite Plioceno-Pleistoceno en todo el mundo.

En el Pozo Cisne No. 1 pudo utilizarse el "datum" Sphaeroidinella para marcar el límite Mioceno-Plioceno, que como ya se discutió antes es evento bioestratigráfico de utilidad mundial. Este "datum" está presente a $2180 \mathrm{~m}$ de profundidad.

La Zona que nos ocupa se encuentra en el Pozo Cisne No. 1 de 950 a $2180 \mathrm{~m}$ de profundidad.

\section{CONCLUSIONES}

\section{CRONOESTRATIGRAFICAS:}

El límite Mioceno-Plioceno pudo ser definido en base a la aparición evolutiva de Sphaeroidinella dehiscens (Parker y Jones).

Los sedimentos que suprayacen al intervalo definido como Plioceno (de 170 a $925 \mathrm{~m}$ ), son considerados de Edad Plioceno-Reciente, unicamente por posición estratigráfica, porque carecen de foraminíferos planctónicos.

\section{BIOESTRATIGRAFICAS:}

Se identificaron dos zonas paleontológicas:

a).- Mioceno Superior:

Zona con Turborotalia acostaensis, presente de 2200 a $4890 \mathrm{~m}$ de profundidad

b).- Plioceno:

Zona con Sphaeroidinella dehiscens, presente de 950 a $2180 \mathrm{~m}$ de profundidad.

De donde se deducen los siguientes espesores:

a). - Para el Mioceno Superior de $2690 \mathrm{~m}$.

b). - Para el Plioceno de $1230 \mathrm{~m}$. 
LAMINA 1

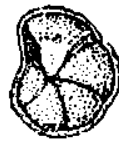

10
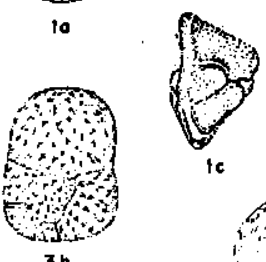

ic

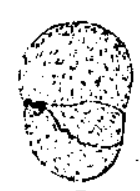

tb

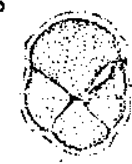

20
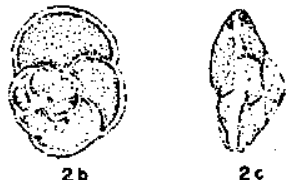

$2 b$

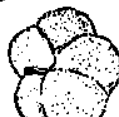

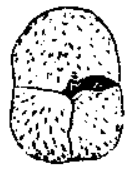

3

$3 b$
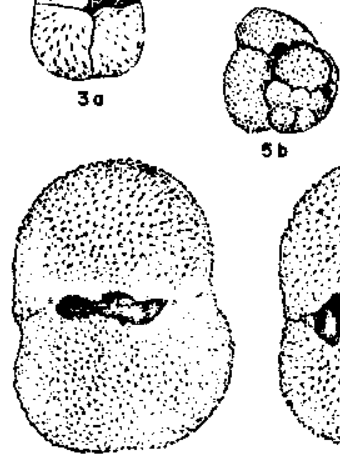

60

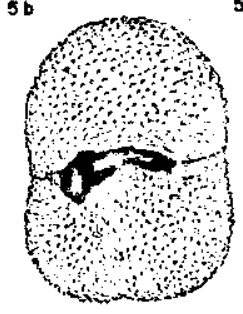

60

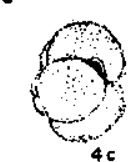
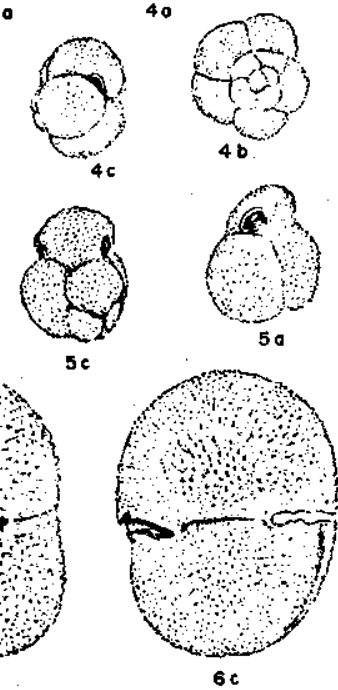

\section{LAMINA 1}

Figs. 18-1c.- Globoratalia ungulata Bermúdez. Pozo Cisne No. 1, 1075 - 1080 m. (X 87). Plioceno.

Figs. 2a-2c.- Globorotalia margaritae Bolli y Bermúdez. Pozo Cisne No. 1, 4600 - 4605 m. (X 91). Mioceno Superior.

Figs. 3a-3c.- Sphaeroidinellopsis subdehiscens Blow. Pozo Cisne No. 1, 2700-2705 m. (X 91). Mioceno Superior.

Figs. 4a-4c.- Turborotalia acostaensis Blow. Pozo Cisne No. 1, 2650 - 2655 m. (X 86). Mioceno Superior.

Figs. 5a-5c.- Globigerinoides obliquus elongatus (d'Orbigny). Pozo Cisne No. 1, 975 - $98 \mathrm{~J}$ m. (X 87). Plioceno.

Figs. 6a-66.- Sphaeroidinella dehiscens (Parker y Jones). Pozo Cisne NG. 1, 1975 - 1980 m. (X 88). Plioceno.

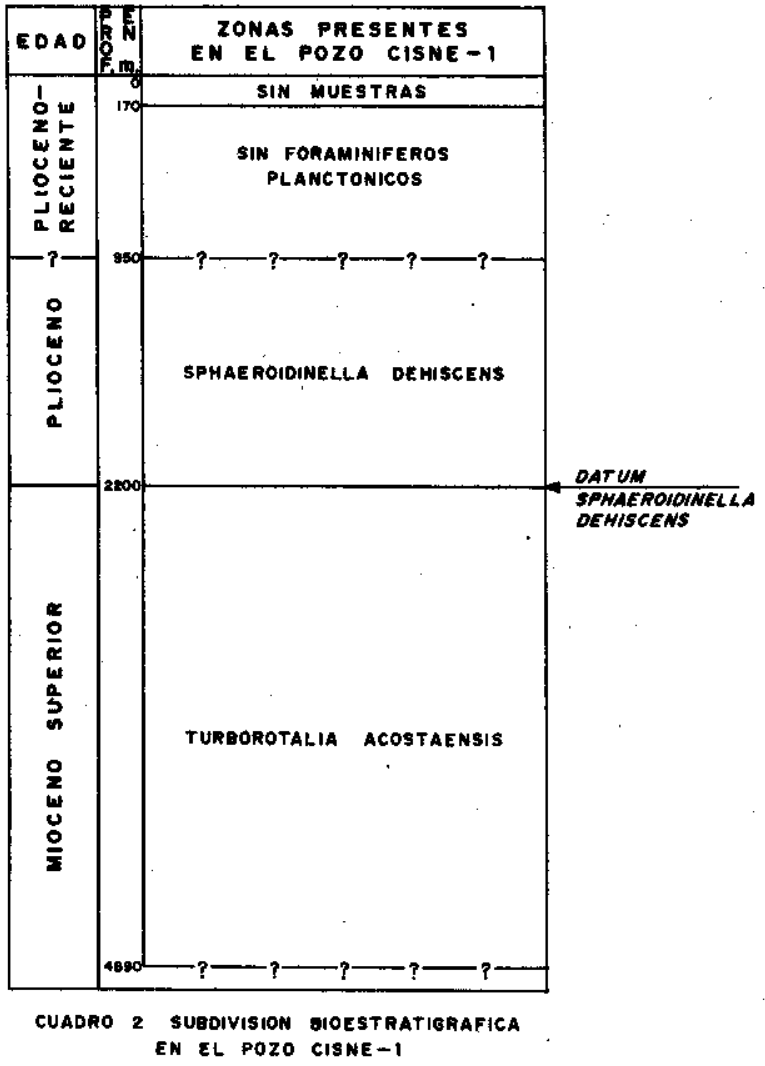




\section{REFERENCIAS BIBLIOGRAFICAS}

ASANO K., TAKAYANAG1. Y TAKAYAMA T., 1975. Late Neogene Epoch boundaries in Japan. In: Saito T. y Burckle L.H. (Eds.) Late Neogene Epoch Boundaries. The American Museum of Natural History. New York, New York, p. 115-123.

BANDY, O.L., 1963a. Aquitanian plaktonic foraminifera from Erben Guyot. Sciencie, vol. 140, No. 3574, p. 1402-1403.

BANDY, O.L., 1963b. Miocene-Pliocene boundary in the Phllippines as related to Late Tertiary stratigraphy of deep-sea sediments. Science, vol. 142, No. 3597, p. 1290-1292.

BANDY, O.L., 1964. Cenozoic planktonic foraminiferal zonation. Micropaleontology, vol. 10, No. 1, p. 1-17.

BANDY, O.L., 1975. Messinian evaporite deposition and the Miocene/Pliocene boundary, Pasquasia Capodarso sections, Sicily. In: Saito T. y Burckle L.H. (Eds.). The American Museum of Natural History. New York, New York, p. 49-63.

BANDY, O.L. Y CASEY, R.E., 1969. Major Late Cenozoic planktonic Datum Planes, Antarctica to the tropics. Antarctic Journal of the United States, vol. 4, No. 5, p. 170-171.

BANDY, O.L. y WADE, M.E., 1969, Miocene-Pleistocene bounderies in deep-water environments. Progress in Oceanography, vol. 4, p. $51-66$.

BANNER, F.T. $\vee$ BLOW, W:H., 1965. Progress in the planktonic foraminiferal biostratigraphy of the Neogene. Nature, vol. 208, p. 1164-1166.

BERGGREN, W.A., 1971. Tertiery boundaries and correlations. In: Funnell and Riedel (Eds.). Micropaleontology of the Oceans. Cambridge Univ. Press. Great Britain, p. 693-809.

BERGGREN, W.A. y VAN COUVERING, J.A., 1974. The Late Neogene: Bioestratigraphy, geochronology and paleoclimatology of the last $\mathbf{1 5}$ miltions years in marine and continental sequences. Paleogeography, Paleoclimatology, Paleoecology, vol. 16, p. $1-216$.

BLOW, W.H., 1959. Age, correlation and biostratigraphy of the upper Tocuyo (San Lorenzo) and Pozon formations, Eastern Falcon, Venezuela. Bulletin of American Peleontology, vol. 39, p. 67-251.

BLOW, W.H., 1969. Late Middle Eocene to Recent planktonic foraminiferal bioestratigraphy. In: Brönnimann, P. and Renz, H. H. (Eds.). Proceedings of the First International Conference of Planktónic Microfossils. Leiden, Netherlands. E.J. Brill, vol. 1, p. 199-421.

CITA, M.B., 1975. The Miocene-Pliocene boundary: History and definition, In: Saito T. y Burckle, L.H. (Eds.). Late Neogene Epoch Boundaries. The American Museum of Natural History. New York, New York, p. 1-30.
CHANG, L., 1975. Miocene-Pliocene boundary in Talwan. In: Saito T., y Burckle L.H., (Eds.). Late Neogene Epoch Boundaries. The American Museum of Natural History. New York, New York, p. $101-105$

HAAK, R. Y POSTUMA, J.A. 1975. The relation between the tropical planktonic foraminifere zonation and the Tertiary Far East letter classification. Geology in Mijnbouw, vol. 51, p. 195-198.

HUANG, T., 1975. Late Neogene foraminiferal zonation of Southwestern Taiwan In: Saito T. y Burckle, L.H. (Eds.) Late Neogene Epoch Bounderles. The Americen Museum of Natural History. New York, New York, p. 106-114.

LYELL, C., 1833. Principles of Geology. John Murray. 1a, Ed. London, vol. 3, 398 pp.

MAYER-EYMAR, K., 1867. Catalogue systématique et descriptif des fossiles des terraines tertiairs qui se trouvent au Muśé Fédéral de Zürich. Zürich Mayer C., vol. 1, p. 1-37; vol. 2, p. $1-65$.

MAYER-EYMAR, K., 1868. Tableau des terrains tertiaires sup6rleurs. Zürich. H. Manz. Ed. 4.

PARKER, F.L. 1964. Foraminifere from the experimental Mohole drilting near Guadalupe Island. Journal of Paleontologv, vol. 38. No. 4, p. 617-636.

PARKER, F.L. 1973. Late Cenozoic bioestratigraphy (planktonic foraminifera) of the Tropical Atlantic deep-sea sections. Reviste Española de Micropaleontologí, vol. 5, No. 2, p. 253289.

SEGUENZA, G., 1868, La formation zanclénne, ou recherches sur une nouvelle formation tertiaire. Soc. Geol. France Bull., ser. 2, vol. 25, P. $465-486$.

SEGUENZA, G., 1879. La foramzioni terziarie nella provincla di Reggio Calabria. Mem. R. Acc. Lincei. Cl. Sc. Fis. Mat, Nat., vol. 3, No. 6, p. $1-446$.

SELLI, R., 1960. II Messiniano Mayer-Eymar, 1867. Proposta di un neostratotipo. Giornale di Geologia, ser. 2, vol. 28, p.1-33

SRNIVASAN, M. S. y SRIVASTAVA, S.S., 1974 Sphaeroidinella dehiscens Datum and Miocene-Pliocene boundary. The American Association of Petroleum Geologists Bulletín, vol. 58, No. 2, p. 304-323.

STAINFORTH, R.M. et al., 1975. Cenozoic planktonic foraminiferal zonation and characteristics of index forms. The University of Kansas Paleontological Contributions Article 62, p. 1425. 